\title{
Radiation Therapy Beam Energy
}

National Cancer Institute

\section{Source}

National Cancer Institute. Radiation Therapy Beam Energy. NCI Thesaurus. Code C152094.

External rays of high energy ionizing radiation that are delivered to precise locations within the body. 